\title{
Physician-augmented pre-hospital care in the Canadian context: the Jasper bus crash mass casualty incident
}

\author{
Brett H. Shaw ${ }^{1,4} \oplus \cdot$ Eddie Chang $^{2,4} \cdot$ Michael Betzner $^{1,4} \cdot$ Jamin Mulvey $^{3,4}$
}

Received: 25 November 2020 / Accepted: 8 April 2021 / Published online: 26 May 2021

() The Author(s), under exclusive licence to Canadian Association of Emergency Physicians (CAEP)/ Association Canadienne de Médecine d'Urgence (ACMU) 2021

Keywords Pre-hospital emergency care $\cdot$ Mass casualty incident $\cdot$ Helicopter emergency medical services $\cdot$ Pre-hospital physician

\section{Introduction}

The role of physicians in Canadian pre-hospital care remains controversial, and in many ways, is in its infancy. Compared to physician-led pre-hospital care models in Europe and Australasia, the Canadian system predominantly utilizes physicians in a remote oversight, coordination, or educational role. There is growing interest in evaluating the potential role of pre-hospital physicians in the Canadian context. Several Canadian Helicopter Emergency Medical Services (HEMS) work with physicians in the pre-hospital environment, attending scene and inter-facility transfers. Here we evaluate the utility of HEMS physicians during the largest mass casualty incident in the history of Jasper National Park.

\section{Case report}

The Columbia Icefield is located within the Canadian Rockies, in Jasper National Park. This site is known for alpine tours, utilizing off-road buses, or snow coaches, to access the glaciers for sightseeing. On July 18th, 2020, a snow coach carrying 27 people left the steep glacier access road, rolling

Brett H. Shaw

brett.shaw@ucalgary.ca

1 Department of Emergency Medicine, University of Calgary, C231 3280 Hospital Dr. NW, Calgary, AB T2N 4Z6, Canada

2 Department of Emergency Medicine, University of Alberta, Edmonton, AB, Canada

3 Department of Anesthesia, University of Calgary, Calgary, $\mathrm{AB}$, Canada

4 Shock Trauma Air Rescue Service (STARS), Calgary, AB, Canada multiple times (Fig. 1). Reports to Emergency Medical Services (EMS) suggested multiple deceased and at least ten critically injured patients.

A massive pre-hospital response ensued over the following 12 hours. National Parks rescue service responded with firefighters and first responders to stabilize and extricate patients. Due to difficult access and steep terrain, 17 patients required heli-sling rescue by rescue helicopter to a staging area, followed by ground transfer 3 kilometres $(\mathrm{km})$ to a casualty collection point. Pre-hospital resources were deployed to the casualty collection point, including 18 ground EMS units, three HEMS, and a commercial helicopter. Staged $120 \mathrm{~km}$ away, five fixed-wing air ambulances were used for rendezvous and secondary patient transfer. The closest trauma centres were $310 \mathrm{~km}$ (Calgary) and $450 \mathrm{~km}$ (Edmonton) away. The closest community hospitals were $100 \mathrm{~km}$ (Jasper) and $185 \mathrm{~km}$ (Banff) away.

Four HEMS physicians played a significant role in the HEMS response. One coordinated the early resource response, destination decisions, and resource allocation offsite by phone. Three on-scene HEMS physicians participated in direct patient care. This included clinical assessment with ultrasound, providing physician orders allowing EMS to deviate from medical control protocols, and performing advanced critical interventions (finger thoracostomies and chest tube insertion).

Three patients were transported by HEMS to trauma centres. 18 additional patients were transported through the coordinated efforts of ground EMS, a commercial helicopter with a HEMS physician onboard, and fixed-wing air ambulance. Transport times to definitive care ranged from 4 to 12 hours. Patients were directed to a mixture of hospitals, depending on injuries. Blunt traumatic injuries included 


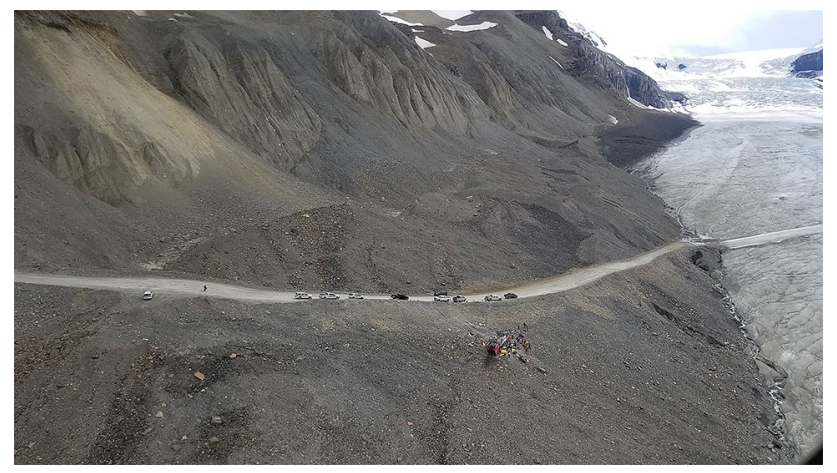

Fig. 1 Incident site of the bus rollover. The Columbia Icefield pictured in the background. Photo taken by Perry Hirsch from Alpine Helicopters in Canmore Alberta

spinal cord injuries, traumatic brain injuries, chest trauma, intra-abdominal bleeding, and orthopaedic injuries.

\section{Discussion}

The pre-hospital phase of care is critical, where the provision of advanced clinical interpretation, diagnostics, and resuscitation can prevent irreversible damage or death from trauma pathology. The benefits of HEMS are to assist with patient access and extrication, minimize time to definitive care, and bring a higher level of care to the patient based on air medical crew training, skill-mix, and decision making [1,2].

Heterogenous models of HEMS configurations exist, including paramedic, nurse-paramedic, and physicianparamedic. Differences in geographical location, team composition, mission profiles, dispatch decisions, quality metrics, and outcomes measures makes research interpretation difficult when discussing evidence for optimum team, skill set, and competencies in pre-hospital care [2].

A physician-augmented HEMS model would seemingly maximize the multidisciplinary care brought to the patient's side. This allows for advanced resuscitation and earlier provision of hospital-based care, reducing the therapeutic vacuum that exists outside of the hospital setting. Literature focused on physician-augmented HEMS models suggest a possible mortality benefit in trauma patients when compared to ground EMS [3, 4]. However, these data are derived from low quality studies with significant heterogeneity [3]. Recent high quality studies directly comparing physician to non-physician HEMS are lacking [5]. Further research is required. In this mass casualty indicent, we believe the provision of HEMS physicians positively impacted patient care and potentially prevented patient deaths. The presence of HEMS physicians allowed for nuanced clinical decision-making, improved triaging, and facilitated advanced interventions outside the scope of non-physician pre-hospital providers.

Nuanced clinical decision-making speaks to the experience physicians have from rigorous in-hospital training. Combining this experience with adequate pre-hospital training allows HEMS physicians to apply this skillset directly to patients in the field. This allows for deviation from the standardized "cookbook" approach to care created when medical control protocols determine scope of practice. This is increasingly necessary with complex critically ill patients requiring resuscitation in a time-dependent fashion. In this mass casualty indicent, there were multiple critical patients with complicated physiological derangements and competing priorities. Working in a multidisciplinary team, HEMS physicians provided leadership in managing these issues including: resuscitation prior to delivering pre-hospital emergency anesthesia; deviation from rapid sequence intubation medical control protocol to avoid worsening peri-intubation hypotension; recognizing simultaneous need for finger thoracostomy and intubation in a patient experiencing respiratory failure, chest trauma, and shock; initiating blood products, vasopressors, and tranexamic acid without delaying to consult online medical control. This physician-augmented pre-hospital care was of particular importance in this wilderness mass casualty indicent, where time to definitive care was prolonged and communications with online medical control were challenging given the remote mountainous terrain.

We believe the HEMS physician skillset was also crucial for patient triage. One physician remained on scene for the duration of the pre-hospital response. They were able to assess each patient using point-of-care ultrasound, influencing pre-hospital treatment decisions (such as tube thoracostomy insertion) and transport modality selection. These clinical assessments were also relayed to the off-site physician coordinating transport decisions, who provided direct clinical updates for incoming patients to receiving trauma team leaders. Traditional mass casualty indicent triage protocols were largely abandoned because of the nuanced clinical assessments the triage physician relayed directly from scene. This allowed patients to be distributed amongst appropriate receiving sites, avoiding overwhelming any one site.

Physician-augmented HEMS systems allow for advanced trauma procedures beyond the critical care paramedic or nurse scope, including finger thoracostomy, resuscitative thoracotomy, lateral canthotomy, and limb amputation [4, 6]. Beyond trauma care, pre-hospital physician delivery to resource-limited centres for inter-facility transport can allow for advanced medical interventions, such as difficult airway management, transvenous pacemaker insertion, linton tube placement, and central venous and arterial line insertion. These interventions represent high acuity, low occurrence events in the pre-hospital context. HEMS physicians have a 
primary hospital-based position, allowing for frequent repetitions in the hospital setting. This enhances their ability to perform procedures quickly and safely in the field.

Overall, the Jasper mass casualty indicent provides a clear example in the Canadian context where physicians with specific pre-hospital training function in an operational HEMS role, bringing specialized hospital-based decisions, interventions, and treatments into an earlier phase of care. This benefit warrants future research in the Canadian system to further define the roles of pre-hospital physicians and physician-augmented HEMS.

\section{Declaration}

Conflict of interest All authors declare they have no conflicts of interest.

\section{References}

1. Michaels D, Pham H, Puckett Y, Dissanaike S. Helicopter versus ground ambulance: review of national database for outcomes in survival in transferred trauma patients in the USA. Trauma Surg Acute Care Open. 2019;4(1):2018-20.

2. Masterson S, Deasy C, Doyle M, Hennelly D, Knox S, Sorensen $\mathrm{J}$. What clinical crew competencies and qualifications are required for helicopter emergency medical services? A review of the literature. Scand J Trauma Resusc Emerg Med. 2020;28(1):1-9.

3. Risgaard B, Draegert C, Baekgaard JS, Steinmetz J, Rasmussen LS. Impact of physician-staffed helicopters on pre-hospital patient outcomes: a systematic review. Acta Anaesthesiol Scand. 2020;64(5):691-704.

4. Maddock A, Corfield AR, Donald MJ, Lyon RM, Sinclair N, Fitzpatrick D, et al. Prehospital critical care is associated with increased survival in adult trauma patients in Scotland. Emerg Med J. 2020;37(3):141-5.

5. Baxt WG, Moody P. The impact of a physician as part of the aeromedical prehospital team in patients with blunt trauma. JAMA J Am Med Assoc. 1987;257(23):3246-50.

6. ter Avest E, Griggs J, Prentice C, Jeyanathan J, Lyon RM. Out-ofhospital cardiac arrest following trauma: what does a helicopter emergency medical service offer? Resuscitation. 2019;135:73-9. 